\title{
Dissipative Particle Dynamics Simulation of Phase Behavior in Bolaamphiphilic Solution*)
}

\author{
Ryōen SHIRASAKI, Yuta YOSHIKAI, Hu-jun QIAN ${ }^{1)}$, Susumu FUJIWARA ${ }^{2)}$, Yuichi TAMURA ${ }^{3)}$ \\ and Hiroaki NAKAMURA ${ }^{4}$ \\ Department of Physics, Faculty of Engineering, Yokohama National University, 79-1 Tokiwadai, Hodogaya-ku, Yokohama \\ 240-8501, Japan \\ ${ }^{1)}$ Institute for Advanced Research and Department of Chemistry, Nagoya University, Nagoya 464-8602, Japan \\ ${ }^{2)}$ Kyoto Institute of Technology, Matsugasaki, Sakyo-ku, Kyoto 606-8585, Japan \\ ${ }^{3)}$ Konan University, 8-9-1 Okamoto, Higashinada-ku, Kobe 658-8501, Japan \\ 4) National Institute for Fusion Science, 322-6 Oroshi-cho, Toki 509-5292, Japan
}

(Received 7 December 2010/ Accepted 12 April 2011)

\begin{abstract}
We study the phase behavior of bolaamphiphilic solution performing the dissipative particle dynamics simulations of coarse-grained bolaamphiphilic molecules with explicit solvent molecules. Our simulations show that there are six kinds of phases: isotropic micellar, micellar, rod-shaped micellar, hexagonal, network-structure and lamellar. The network-structure and the lamellar phases disappear when the restoring potential against the bending of bolaamphiphilic molecules in our simulation model is excluded; and the isotropic micellar and the hexagonal phases disappear when the restoring potential is included. This suggests that the bending potential is important in the formation of the higher-ordered structures by the bolaamphiphilic molecules.
\end{abstract}

(C) 2011 The Japan Society of Plasma Science and Nuclear Fusion Research

Keywords: bolaamphiphilic solution, dissipative particle dynamics simulation, phase diagram, micellar phase, hexagonal phase, lamellar phase

DOI: $10.1585 /$ pfr.6.2401116

\section{Introduction}

Investigation of self-organization in nonequilibrium and nonlinear systems is very important to improve plasma confinement. To gain insights into the universal self-organizing properties in nature, we study the self-organization in model systems such as amphiphilic molecules. Amphiphilic molecules such as lipids and surfactants contain hydrophilic and hydrophobic parts. In aqueous solvents, amphiphilic molecules often selfassemble spontaneously into various structures such as micelles, mesophases, and bicontinuous cubic structures [1]. The phase behavior of coarse-grained amphiphilic dimers in solution has been investigated by molecular dynamics simulations [2] and by dissipative particle dynamics (DPD) simulations [3,4]. Although several experimental studies on the supramolecular architectures based on bolaamphiphilic molecules, containing a hydrophobic stalk and two hydrophilic ends, have been done [5], there have only been a few theoretical and simulation studies on the phase behavior of bolaamphiphilic solutions. The purpose of this study is to clarify the phase behavior of bolaamphiphilic solutions. With a view to investigating the phase behavior of bolaamphiphilic solutions at the molecular level, we perform DPD simulations on coarse-grained bolaamphiphilic

author's e-mail: sirasaki@ynu.ac.jp

*) This article is based on the presentation at the 20th International Toki Conference (ITC20). molecules with explicit solvent molecules and analyze the formation processes of micelles and mesophases.

\section{Simulation Method}

\subsection{DPD algorithm}

First, we express the DPD model and algorithm [3,6]. According to ordinary DPD model, all atoms are coarsegrained to particles whose masses are the same. We define the total number of particles as $N$. The position and velocity vectors of particle $i(i=1, \cdots, N)$ are indicated by $\vec{r}_{i}$ and $\vec{v}_{i}$, respectively. Particle $i$ moves according to the following equations of motion, where all physical quantities are made dimensionless.

$$
\frac{\mathrm{d} \vec{r}_{i}}{\mathrm{~d} t}=\vec{v}_{i}, \quad m \frac{\mathrm{d} \vec{v}_{i}}{\mathrm{~d} t}=\sum_{j(\neq i)}^{N} \vec{F}_{i j}+\sum_{\langle j, k\rangle(j, k \neq i)} \vec{F}_{i, j, k},
$$

where $m$ is the mass of each particle and $\langle j, k\rangle$ denotes the summation over combinations of $j$ and $k . \vec{F}_{i j}$ is the total force between two particles which acts on particle $i$ from particle $j . \quad \vec{F}_{i, j, k}$ is a force from a three-body interaction which acts on particle $i$ from a combination of particles $j$ and $k . \vec{F}_{i j}$ is written as follows:

$$
\vec{F}_{i j}=\vec{F}_{i j}^{\mathrm{C}}+\vec{F}_{i j}^{\mathrm{R}}+\vec{F}_{i j}^{\mathrm{D}}+\vec{F}_{i j}^{\mathrm{B}} .
$$

In Eq. $2, \vec{F}_{i j}^{\mathrm{C}}$ is a conservative force deriving from a potential exerted on particle $i$ by particle $j, \vec{F}_{i j}^{\mathrm{D}}$ and $\vec{F}_{i j}^{\mathrm{R}}$ are the 
dissipative and random forces between particles $i$ and $j$, respectively, and $\vec{F}_{i j}^{\mathrm{B}}$ is the bond-stretching force between neighboring particles.

The conservative force $\vec{F}^{\mathrm{C}}$ has the following form:

$$
\vec{F}_{i j}^{\mathrm{C}}=-\nabla_{i} \phi_{i j},
$$

where $\nabla_{i} \equiv \partial / \partial \vec{r}_{i}$. For computational convenience, we adopted the cut-off length as the unit of length. It is assumed that the conservative force $\vec{F}^{\mathrm{C}}$ are truncated at a radius of this length. Following this assumption, the twopoint potential $\phi_{i j}$ in Eq. 3 is defined as follows:

$$
\phi_{i j} \equiv \phi\left(r_{i j}\right)=\frac{1}{2} a_{i j}\left(r_{i j}-1\right)^{2} H\left(1-r_{i j}\right)
$$

where $r_{i j}=\left|\vec{r}_{i j}\right| ; \vec{r}_{i j} \equiv \vec{r}_{j}-\vec{r}_{i}$. We also define the unit vector $\vec{n}_{i j} \equiv \vec{r}_{i j} / r_{i j}$ between particles $i$ and $j$. The step function $H$ in Eq. 4 is defined by

$$
H(x) \equiv \begin{cases}0 & \text { for } x<0 \\ 1 & \text { for } x \geq 0\end{cases}
$$

Español and Warren proposed the following simple forms for the random and dissipative forces [7]:

$$
\begin{aligned}
& \vec{F}_{i j}^{\mathrm{R}}=\sigma \omega_{\mathrm{R}}\left(r_{i j}\right) \vec{n}_{i j} \frac{\zeta_{i j}}{\sqrt{\Delta t}}, \\
& \vec{F}_{i j}^{\mathrm{D}}=-\gamma \omega_{\mathrm{D}}\left(r_{i j}\right)\left(\vec{v}_{i j} \cdot \vec{n}_{i, j}\right) \vec{n}_{i j},
\end{aligned}
$$

where $\omega_{R}$ and $\omega_{D}$ are introduced as the weight functions of random and dissipative forces, respectively. $\zeta_{i j}$ is a Gaussian random variable with zero mean and unit variance, chosen independently for each pair $(i, j)$ of interacting particles at each time step, and $\zeta_{i j}=\zeta_{j i} . \vec{n}_{i j}$ is the unit vector between particles $i$ and $j$. The strength of the dissipative and random forces is determined by the dimensionless parameters $\sigma$ and $\gamma$, respectively. The parameter $\Delta t$ is a dimensionless time interval of integrating the equation of motion.

Now we consider the fluctuation-dissipation theorem of the DPD method. The time evolution of the distribution function of the DPD system is governed by the Fokker-Planck equation [7]. The system evolves to the same steady state as the Hamiltonian system, that is, the Gibbs-Boltzmann canonical ensemble, if the coefficients of the dissipative and random force terms have the following relations:

$$
\omega_{\mathrm{D}}=\left(\omega_{\mathrm{R}}\right)^{2}, \quad \sigma^{2}=2 T \gamma,
$$

where $T$ is the dimensionless equilibrium temperature. The forms of the weight functions $\omega_{\mathrm{D}}$ and $\omega_{\mathrm{R}}$ are not specified in the original DPD algorithm. We adopted a simple form of the weighting function $\omega_{\mathrm{R}}(r)=\omega_{\mathrm{D}}^{1 / 2}(r)=\omega(r)$, where the function $\omega$ is defined by $\omega(x) \equiv(1-x) H(1-x)$. $[6,7]$

Finally, we introduce the dimensionless potential energies $\phi_{i j}^{\mathrm{B}}$ and $\phi_{i j k}^{\mathrm{BM}} \cdot \phi_{i j}^{\mathrm{B}}$ corresponds to the bond-stretching force $\vec{F}_{i j}^{\mathrm{B}}$. If particle $i$ is connected to particle $j, \phi_{i j}^{\mathrm{B}}$ is
Table 1 Coefficients $a_{i j}$, which depend on kinds of particles $i$ and $j$.

\begin{tabular}{|c|ccc|}
\hline$a_{i j}$ & $\mathrm{~W}$ & $\mathrm{~A}$ & $\mathrm{~B}$ \\
\hline $\mathrm{W}$ & 25 & 25 & $\alpha$ \\
$\mathrm{A}$ & 25 & 25 & $\alpha$ \\
$\mathrm{B}$ & $\alpha$ & $\alpha$ & 25 \\
\hline
\end{tabular}

NOTE: $\mathrm{W}$ is a "water" particle, A is a "hydrophilic" particle and $\mathrm{B}$ is a "hydrophobic" particle. $\alpha$ is varied from 50 to 90 in simulations.

given by $\phi_{i j}^{\mathrm{B}}=\phi^{\mathrm{B}}\left(r_{i j}\right)=a_{\mathrm{B}} r_{i j}^{2} / 2$, otherwise $\phi_{i j}^{\mathrm{B}}=0$, where $a_{\mathrm{B}}$ is the potential energy coefficient. $\phi_{i j k}^{\mathrm{BM}}$ is the potential energy that causes $\vec{F}_{i, j, k}$. For ABA molecules, $\vec{F}_{i, j, k}$ is the restoring force against bending of the ABA axis. If particle $i$ is ' $\mathrm{A}^{\prime}$ and is connected to a combination of particle $j$ ('B') and particle $k$ ('A'), $\phi_{i j k}^{\mathrm{BM}}$ is given by $\phi_{i j k}^{\mathrm{BM}}=\phi^{\mathrm{BM}}\left(\vec{r}_{i j}, \vec{r}_{j k}\right)=a_{\mathrm{BM}}\left\{\cos ^{-1}\left(\left|\vec{r}_{i j} \cdot \vec{r}_{j k}\right| /\left(r_{i j} r_{j k}\right)\right)\right\}^{2} / 2$ otherwise $\phi_{i j k}^{\mathrm{BM}}=0$, where $a_{\mathrm{BM}}$ is the potential energy coefficient.

\subsection{Simulation model and parameters}

In our simulation, we use modified Jury model molecule that is composed of a hydrophilic particle (A) and a hydrophobic particle (B) [3]. Water molecules are modeled as particles $\mathrm{W}$. The mass of all particles are assumed to be unity. The number density of particles $\rho$ is set to $\rho=5$. Total number of particles $N \equiv 3 N_{\mathrm{ABA}}+N_{\mathrm{W}}$ is fixed to $N=5000$, where $N_{\mathrm{ABA}}$ is the number of modeled bolaamphiphilic molecules ABA and $N_{\mathrm{W}}$ is the number of water particles.

The simulation box is set to cubic. The dimensionless length of the box $L$ is $L=(N / \rho)^{1 / 3}=10.0$. We use periodic boundary conditions in simulation. The interaction coefficients $a_{i j}$ in Eq. 4 are presented in Table 1. The coefficient of the interaction between $\mathrm{A}$ and $\mathrm{B}$ is written by a variable $\alpha$ which is varied from 50 to 90 in our simulation. The strength of the interaction between $\mathrm{W}$ and $\mathrm{B}$ is set to be equal to that of the interaction between $\mathrm{A}$ and $\mathrm{B}$. The coefficient of the bond-stretching potential $a_{\mathrm{B}}$ is adopted as $a_{\mathrm{B}}=100$. We use the dimensionless time-interval as $\Delta t=0.06$. The dimensionless strength of the random and dissipative forces are $\sigma=3.0$ and $\gamma=9.0 /(2 T)$, respectively.

In the initial configuration, all molecules and water particles are located randomly and the velocity of each particle is set under Maxwell distributions with dimensionless temperature $T$. We prepare ABA solutions of various concentrations $\left(0.1 \leq c=3 N_{\mathrm{ABA}} / N \leq 0.9\right)$ and DPD simulations of $1.0 \times 10^{7}$ time intervals are carried out for each run.

\section{Simulation Results and Discussion 3.1 Self-assembled structures for $a_{\mathrm{BM}}=\mathbf{0}$}

First, we set the interaction parameter $a_{\mathrm{BM}}$ to $a_{\mathrm{BM}}=0$. 


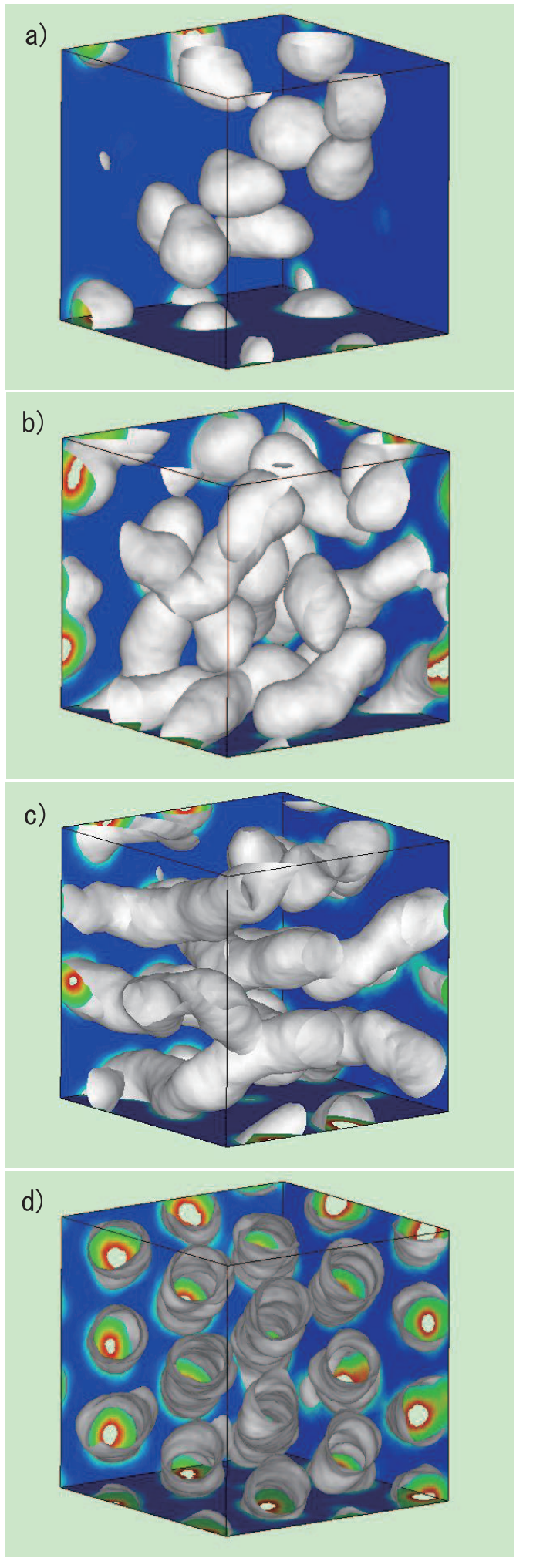

Fig. 1 Self-assembled structures of the bolaamphiphilic molecules in the case of $T=1.0$ and $\alpha=70$ for various concentrations $c$. The interaction parameter $a_{\mathrm{BM}}$ of the bending potential is set to $a_{\mathrm{BM}}=0$. The isosurfaces of density of the hydrophobic particles (i.e., B particles) are shown. a) The isotropic micellar phase $(c=0.2)$, b) the micellar phase $(c=0.3), \mathrm{c})$ the rod-shaped micellar phase $(c=0.6)$, and $d)$ the hexagonal phase $(c=0.8)$.
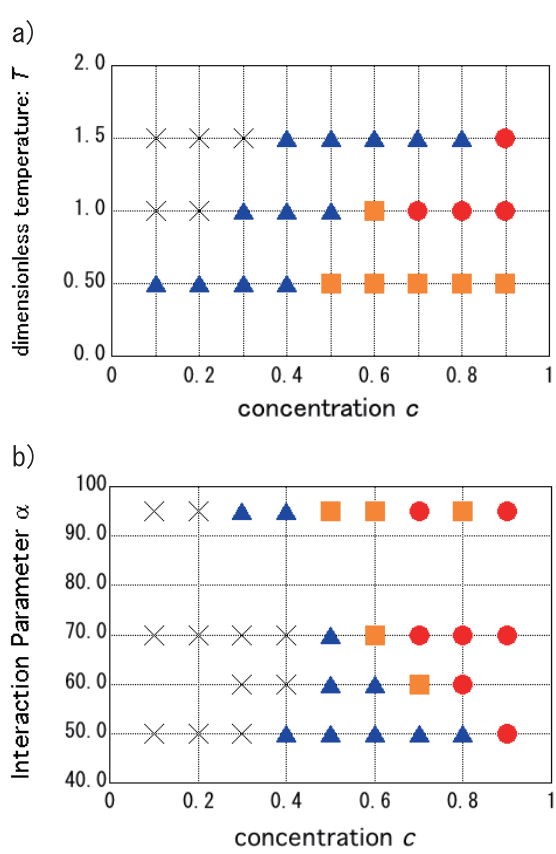

Fig. 2 (Color online) Phase diagrams. The interaction parameter $a_{\mathrm{BM}}$ is set to $a_{\mathrm{BM}}=0$. a) Dimensionless temperature $T$ vs. concentration phase diagram of ABA solution. b) Intensity of the interaction coefficient $\alpha$ vs. concentration phase diagram of ABA solution. In these figures, the abscissa represents the concentration $c$. Symbol $\times$, blue triangles, brown squares, and red circles represent the isotropic micellar phase, the micellar phase, the rodshaped micellar phase, and the hexagonal phase, respectively.

This excludes the effect of the bending potential energy. The dimensionless temperature $T$ and the parameter $\alpha$ are selected to be 1.0 and 70, respectively. Figure 1 shows the self-assembled structures of molecules at various concentrations $c=0.2,0.3,0.6$, and 0.8 . The isosurfaces of the densities of the hydrophobic particles (i.e., B particles) are shown. An isotropic micellar phase (i.e., the phase in which the globe-shaped micelles mainly appear) is observed at low concentration $(c=0.2)$, whereas a hexagonal phase is observed at high concentration $(c=0.8)$. These figures tell us that, as the concentration $c$ increases, the self-assembled structures of the molecules grow and they form higher-ordered structures.

\subsection{Phase diagram for $a_{\mathrm{BM}}=0$}

We simulated the other cases of $(\alpha, c, T)$ to obtain the phase diagram of ABA molecule in $\mathrm{W}$ monomers. (see Fig. 2) In Fig. 2a), the ordinate denotes the dimensionless temperature $T$ and the interaction coefficient $\alpha$ is selected to be 70 . In Fig. 2b), the ordinate denotes the intensity of the interaction coefficient $\alpha$. The dimensionless temperature $T$ is set to $T=1.0$. The abscissas of these figures represent the concentration $c$. The obtained molecular configurations are classified into four phases: the isotropic micellar, the micellar, the rod-shaped micellar, and the 

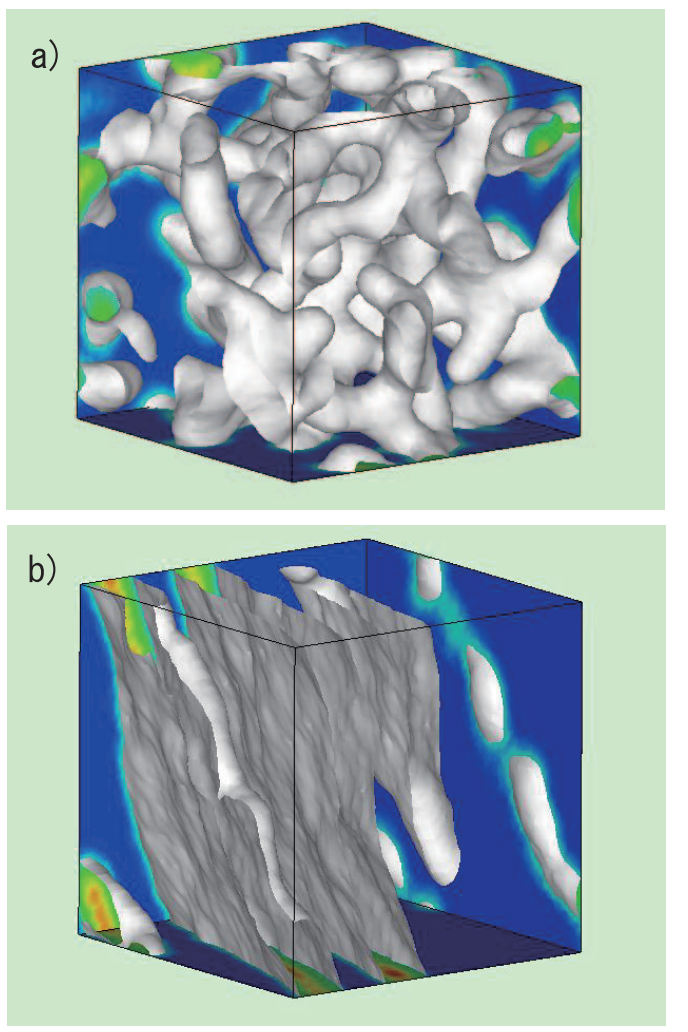

Fig. 3 The self-assembled structure of the bolaamphiphilic molecules when $a_{\mathrm{BM}}=10.0, c=0.5$, and $T=1.0$. Isosurfaces of densities of the hydrophobic particles are shown. a) The network-structure phase $(\alpha=50)$. b) The lamellar phase $(\alpha=70)$.

hexagonal.

\subsection{Self-assembled structures and phase di- agrams for $\boldsymbol{a}_{\mathrm{BM}} \neq 0$}

Figure 3 shows the self-assembled structures obtained when $a_{\mathrm{BM}}=10.0$, and the dimensionless temperature $T$ and the concentration $c$ are selected to be 1.0 and 0.5 , respectively. A network-structure phase and a lamellar phase are formed at $\alpha=50$ and 70, respectively.

We simulated the other cases of $(\alpha, c)$ to obtain phase diagram in which $a_{\mathrm{BM}}$ and $T$ are set to $a_{\mathrm{BM}}=10.0$ and $T=1.0$, respectively. The obtained molecular configurations are classified into four phases: the micellar, the rodshaped micellar, the network-structure, and lamellar. In Fig. 4, the simulated phase diagram is shown. The ordinate denotes the intensity of the interaction coefficient $\alpha$ and the abscissas of these figures represent the concentration $c$. The network-structure phase is observed at high concentrations when $\alpha=50$ whereas the lamellar phase is observed when $\alpha \geq 55$.

\section{Conclusions}

We have performed DPD simulations of bolaamphiphilic solutions. We found that four kinds of phases (isotropic micellar, micellar, rod-shaped micellar, and

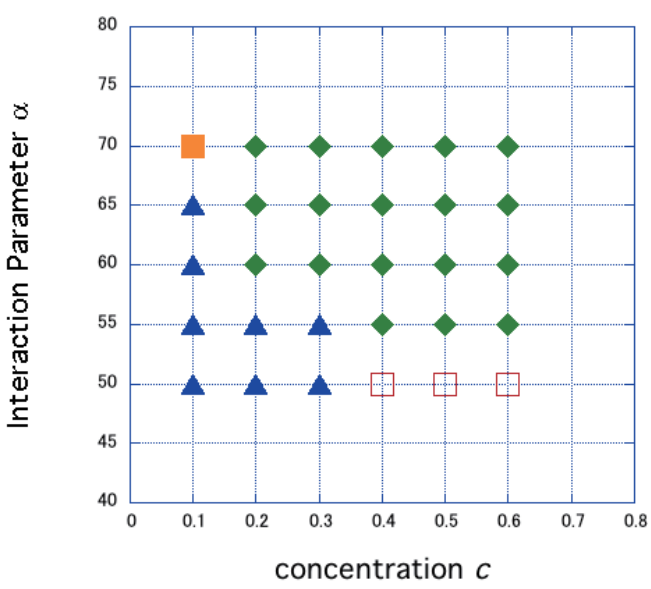

Fig. 4 (Color online) Phase diagram of ABA solution. $a_{\mathrm{BM}}$ and $T$ are set to $a_{\mathrm{BM}}=10.0$ and $T=1.0$, respectively. The ordinate denotes the intensity of the interaction coefficient $\alpha$ and the abscissa represents the concentration $c$. Blue triangles, brown squares, open squares, and green rhombuses represent the micellar phase, the rod-shaped micellar phase, the network-structure phase, and the lamellar phase, respectively.

hexagonal phase) were formed when $a_{\mathrm{BM}}=0$. When $T \geq 1.0$, the isotropic micellar phase is observed at lower concentrations, whereas the hexagonal phase is observed at high concentrations. In the case of $a_{\mathrm{BM}}=10.0$, two new phases appear, namely, the network-structure phase and the lamellar phase. The isotropic micellar phase and the hexagonal phase disappear. The phase behavior of the bolaamphiphilic solutions is obtained for $a_{\mathrm{BM}}=0$ and $a_{\mathrm{BM}}=10.0$ in Figs. 2 and 4, respectively. It would be very interesting to know if these phase behaviors can be observed experimentally. A detailed examination is future problem.

This work is supported by Grants-in-Aid for Scientific Research (C) [No. 22560004] and Scientific Research (C) [No. 22540425] as well as the NIFS Collaboration Research program (NIFS09KDBN002) and the NINS undertaking Forming Bases for Interdisciplinary and International Research through Cooperation Across Fields of Study and Collaborative Research Programs (Nos. NIFS09KEIN0091 and NIFS10KEIN0161).

[1] I.W. Hamley, Introduction to Soft Matter (J. Wiley, Chichester, Rev. ed., 2007).

[2] S. Fujiwara, D. Funaoka, T. Itoh and M. Hashimoto, Comput. Phys. Commun. 182, 192 (2011).

[3] S. Jury, P. Bladon, M. Cates, S. Krishna, M. Hagen, N. Ruddock and P. Warren, Phys. Chem. Chem. Phys. 1, 2051 (1999).

[4] H. Nakamura, Mol. Simulation 30, 941 (2004).

[5] T. Shimizu, M. Masuda and H. Minamikawa, Chem. Rev. 105, 1401 (2005).

[6] R.D. Groot and P.B. Warren, J. Chem Phys. 107, 4423 (1997).

[7] P. Español and P. Warren, Europhys. Lett. 30, 191 (1995). 